Amer, J. Bot. 64(6): 791-798. 1977.

\title{
CHROMOSOME COUNTS OF COMPOSITAE FROM MEXICO AND THE UNITED STATES ${ }^{1}$
}

\author{
David J. KeIL And Tod F. Stuessy \\ Department of Biological Sciences, California Polytechnic State University, San Luis Obispo \\ 93407, and Department of Botany, The Ohio State University, Columbus 43210

\section{A B S T R A C T} \\ Chromosome counts of Compositae are reported from Mexico and the United States. First \\ generic counts are from Egletes $(n=27)$, Hydropectis $(n=9)$, and Pippenalia $(2 n=$ ca. 60$)$. \\ First counts also are reported for 22 specific and infraspecific taxa in Baccharis, Erigeron, \\ Flaveria, Gnaphalium, Grindelia, Helenium, Heterotheca, Melampodium, Montanoa, Pery- \\ menium, Piqueria, Senecio, Stevia, Verbesina, Xanthocephalum, and Zaluzania. Additional \\ counts also are provided for 123 populations of taxa counted previously, of which eight are new \\ numbers. Taxonomic implications of certain counts are discussed.
}

THIS PAPER is a continuation of our studies (Keil and Stuessy, 1975) on chromosome numbers in the Compositae principally from the western United States and Mexico. As before, we have adopted the policy of reporting new populational counts of previously documented taxa as well as first reports for genera, species and varieties. The materials and methods involving conventional acetocarmine squashes of buds for meiotic stages are the same as those mentioned in our earlier paper. All voucher specimens are on deposit in the herbarium of the Ohio State University (OS).

RESULTS-The chromosome counts obtained are listed in Table 1. First counts are reported for three genera, 20 species, and two varieties; 123 additional counts are for taxa counted previously, eight of which are new numbers. The first counts for genera are from Egletes Cass. ( $n$ = 27), Hydropectics Rydb. $(n=9)$, and Pippenalia McVaugh $(2 n=$ ca. 60$)$, and first counts for species are in Baccharis L., Erigeron L., Flaveria Juss., Gnaphalium L., Grindelia Willd., Helenium L., Heterotheca Cass., Melampodium L., Montanoa Cav., Perymenium Schrad., Piqueria Cav., Senecio L., Stevia Cav., Verbesina L., Xanthocephalum Willd., and Zaluzania Pers.

DISCUSSION-As in our earlier paper (Keil and Stuessy, 1975), the discussions are restricted to

\footnotetext{
${ }^{1}$ Received for publication 30 September 1976; revision accepted 8 February 1977.

Field work for this study was completed under NSF grants GB-30240 and GB-37678. Thanks go to: Marvin Roberts and Robert Gardner for assistance during collecting trips; Rogers McVaugh of the University of Michigan and Lorin Nevling of the Field Museum for courtesy and assistance during our research visits to their herbaria; Ted Barkley and Mike Powell for the identification of several of our collections of Senecio, Flaveria and Perityle; and Loran Anderson for permission to include an unpublished mitotic count of Melampodium repens.
}

first or new counts or significant comments. The order of commentary follows the sequence of tribes in the classification of Hoffmann (1890-94; with the additional recognition of the Tageteae as a separate tribe [Rydberg, 1915-16]), which is the same as that used in Table 1. References for statements regarding the broad range of chromosomal variation within genera will not be given; documentation for these counts comes from available chromosomal indices (Darlington and Wylie, 1955; Cave, 1958-65; Ornduff, 1967-69; Fedorov, 1969; Moore, 1970-74).

A number of first reports for taxa are consistent with previous counts for the respective genera. These are listed here and will not be discussed further: Baccharis heterophylla, $n=9$; Erigeron dryophyllus, $n=9$; Flaveria robusta, $n$ $=18$; Gnaphalium cf. chartaceum and G. stramineum, $n=14$; Grindelia tenella, $n=6$; Heterotheca chrysopsidis, $n=9 ;$ Montanoa pyramidata, $n=19$; Senecio arizonicus, S. flaccidus, and $S$. wootonii, $n=20$; Stevia aschenborniana, $n=11$; $S$. berlandieri A. Gray var. berlandieri, $n=12$; Verbesina tequilana, $n=17$; and Zaluzania discoidea, $n=18$.

Eupatorieae-Our count of ca. 84 I for an undetermined species of Ageratina is indicative of the cytological complexity of this genus. Based upon previous reports at the diploid, tetraploid, and apparently pentaploid levels, King and Robinson (1970) postulated a base number of $x=$ 17 for Ageratina. Our count probably represents a pentaploid $(5 x=85)$. Insight into the biological complexity of Ageratina is hindered by the lack of any published revision of the Latin American taxa. King and Robinson (1970) listed over 200 species for the genus (most as transfers from Eupatorium L.) but provided no keys, descriptions, or other means of identification.

Our count of $n=20$ for Eupatorium sagitta- 
tum is the first polyploid count for this species. Diploid counts of $n=10$ have been reported previously (Turner and Flyr, 1966; Keil and Pinkava, 1976), also from Sinaloa where this taxon is common (Robinson, 1926).

Five species of Piqueria have been counted before as $n=10,11,12$, and 24. Our first report of $n=12$ for $P$. cf. triflora conforms to the known chromosomal diversity. Our voucher differs from the typical $P$. triflora in having glabrous rather than hirtellous phyllaries and stems.

Astereae-Achaetogeron, a genus of about eight species, has had two chromosomal levels reported previously: $n=9$ from $A$. pinnatifidum A. Gray (Turner and Flyr, 1966) and A. griseus Greenm. (DeJong and Longpre, 1963); and $n$ $=27$ from $A$. forreri Greene (DeJong and Longpre, 1963). Our count of $n=36$ for $A$. cf. forreri is not only a new chromosomal level for this species, but also the first report of the octoploid level for the genus.

Our count of $n=27$ for Egletes viscosa is the first for the genus. An ancestral base number of $x=9$ for Egletes is suggested, especially because two related genera (Hoffmann, 1890-94; Shinners, 1949), Grangea Adans. and Dichocephala DC., are known also as $n=9$.

Our two populational counts of $n=8$ for Gutierrezia glutinosa are the first reports of a tetraploid chromosomal level for this species, which has been counted before as $n=4$ (e.g., Powell and Turner, 1963; Reveal and Spellenberg, 1976). Infraspecific euploidy is not uncommon in Gutierrezia, and it has been reported (Solbrig, 1960a) from G. bracteata Abrams ( $n$ $=8$ and 12), G. microcephala (DC.) A. Gray ( $n=8$ and 16), and G. sarothrae (Pursh) Britt. $\&$ Rusby ( $n=4$ and 8$)$.

Two chromosomal base numbers are known in Xanthocephalum: $x=4$ and 6 . Our first report of $n=6$ for $X$. benthamianum fits well with its close morphological relationship to $X$. gymnospermoides (A. Gray) B. \& H. (Solbrig, 1961) which is also $n=6$ (e.g., Solbrig, 1960b). However, $X$. benthamianum also bears a strong overall morphological resemblance to some species of Grindelia $(x=6)$. The possibility that the species of Xanthocephalum with a base of $x=6$ are more closely related to Grindelia than to the $x$ $=4$ species of Xanthocephalum needs to be examined critically.

Heliantheae-Many chromosome counts have been made in Bidens, and the genus is clearly on a base of $x=12$. Bidens reptans var. urbanii has been counted before at the tetraploid level of $n=$ 24. (Turner, Ellison, and King, 1961). Our new count of $n=12$ indicates the presence of infraspecific euploidy. Two populational counts of $n$ $=11$ also have been reported for $B$. cf. reptans (Powell and Turner, 1963).
Counts have been published previously for 27 of the 37 recognized species of Melampodium (Stuessy, 1970, 1971; Keil and Stuessy, 1975). Of particular interest, therefore, are the first chromosome counts for two additional species, $M$. repens $(2 n=54)$ and $M$. tepicense $(n=12)$. The former taxon and the related $M$. bibracteatum S. Wats. constitute section Bibractiaria (Steussy, 1971), which has been the only section of the genus still unknown cytologically. The relationships of this section with other sections are difficult to assess due to the extreme reduction of floral features in both species. The chromosome number of $2 n=54$ seems probably based on $x=$ 9 , which would place this group closest to section Zarabellia with the same chromosomal base (counts of $n=9,18$, and 27 have been reported). Morphologically, $M$. longifolium Cerv. ex Cav. would be the species possibly linking the two sections, although the connection is distant. The high ploidy level (probably hexaploid) of $M$. repens corresponds with its specialized prostrate growth habit and reduced floral features. On morphological grounds, $M$. tepicense is most closely related to $M$. divaricatum (Rich. in Pers.) DC. and $M$. dicoelocarpum Robins. of section Serratura (Stuessy, 1972). The first chromosome report for $M$. tepicense of $n=12$ fits well with the sectional base number of $x=12$ and corroborates the placement of the species on morphological criteria.

Our two chromosome counts of $n=18$ for Parthenice mollis confirm two recent reports from this species of the same number (Urbatsch, 1975; Sauck, 1975). The firm establishment of a chromosome number for the monotypic Parthenice is of particular interest to the junior author, because in a previous paper (Stuessy, 1973) the genus was transferred from the subtribe Melampodiinae to the Ambrosiinae near Parthenium based on overall morphological resemblance. The chromosome counts of $n=18$ for Parthenice fit well with the presumptive base of $x=18$ for the entire Ambrosiinae (Payne, Raven, and Kyhos, 1964). Recent pollen studies on Parthenice (Bolick and Skvarla, 1976) do not contradict this placement in the Ambrosiinae.

The previous chromosome counts $(n=15,30$, ca. 45 , ca. 86) in Perymenium are based clearly on $x=15$. Our first report of $n=30$ for $P$. buphthalmoides (var. tenellum) suggests a tetraploid species. Two previous counts of $n=15$ (Turner, Beaman, and Rock, 1961) and $n=45$ (Strother, 1976) have been reported for Perymenium mendezii. Our count of $n=30$ completes the euploid series within this taxon. Perymenium nelsonii Rob. \& Greenm. is the only other species known to have infraspecific euploidy ( $n$ $=15$ and ca. 86; Turner, Ellison, and King, 1961; Solbrig, Kyhos, Powell, and Raven, 1972).

Verbesina crocata has been counted previously 
TABLE 1. Chromosome counts of Compositae from Mexico and the United States

\begin{tabular}{ccc}
\hline \hline Taxon & Gametic chromosome number & Voucher \\
\hline
\end{tabular}

\section{EUPATORIEAE}

${ }^{+}$Ageratina sp.

Brickellia glomerata Fern.

Eupatorium greggii A. Gray

${ }^{+}$Eupatorium sagittatum A. Gray

**Piqueria cf. triflora Hemsl.

**Stevia aschenborniana Sch. Bip.

* Stevia berlandieri A. Gray var. berlandieri

Stevia elongata H.B.K.

ca. $35 \mathrm{I}^{\mathrm{a}}$

Stevia eupatoria (Spreng.) Willd.

ca. $33 \mathrm{I}^{\mathrm{a}}$

Stevia origanoides H.B.K.

Stevia salicifolia Cav.

Stevia subpubescens Lag.

Stevia trifida Lag.

Stevia sp.

\section{ASTEREAE}

${ }^{+}$Achaetogeron cf. forreri Greene

Astranthium orthopodum (Robins. \& Fern.)

Larsen

**Baccharis heterophylla H.B.K.

Baccharis sordescens DC.

Conyza coronopifolia H.B.K.

Conyza coulteri A. Gray

***Egletes viscosa (L.) Less var. dissecta Shinners

Erigeron cf. coronarius Greene

**Erigeron dryophyllus A. Gray

Grindelia cf. inuloides Willd.

**Grindelia tenella Steyerm.

${ }^{+}$Gutierrezia glutinosa (Schauer) Sch. Bip.

Gymnosperma glutinosum (Spreng.) Less.

**Heterotheca chrysopsidis DC.

Heterotheca villosa (Pursh) Shinners

Heterotheca villosa (Pursh) Shinners

Machaeranthera boltoniae (Greene) Turner \&

Horne

Solidago velutina DC.

**Xanthocephalum benthamianum Hemsl.

\section{INULEAE}

Gnaphalium cf. attenuatum DC.

**Gnaphalium cf. chartaceum Greenm.

Gnaphalium purpureum L. ca. $84 \mathrm{I}^{\mathrm{a}} \quad$ MEXICO: Durango: $1 \mathrm{mi}$ NE of Los Angeles, $R K$ $10246^{\mathrm{b}}$

9 MEXICO: Michoacán: $9.7 \mathrm{mi} \mathrm{S}$ of Uruapan, $S R 3750^{\circ}$

10 MEXICO: Coahuila: $53 \mathrm{mi} \mathrm{E}$ of turnoff to San Pedro on rte $40, R K 10415$. Durango: jct rtes $45 \& 30$, $R K 10405$.

20 MEXICO: Sinaloa: $37 \mathrm{mi}$ SE of Culiacán, $K 8803 A^{\mathrm{d}}$

12 MEXICO: Sinaloa: Loberas, $R K 10202$.

11 MEXICO: México: ca. $19 \mathrm{mi}$ SE of Zitacuaro, $S R$ 3718.

12 MEXICO: Nuevo León: Chipinque Mesa, $R K$ 10424; $26 \mathrm{mi} \mathrm{W}$ of Linares, $R K 10474$.

MEXICO: México: ca. $19 \mathrm{mi} \mathrm{SE}$ of Zitácuaro, $S R$ 3714.

MEXICO: Durango: Río Mimbres at rte 40, $R K$ 10351.

11 MEXICO: Durango: $11.5 \mathrm{mi} \mathrm{SW}$ of La Ciudad, $R K$ 10261.

12 MEXICO: Durango: $5 \mathrm{mi} \mathrm{W}$ of Durango, $R K 10336$.

12 MEXICO: Michoacán: ca. $8 \mathrm{mi} \mathrm{SE}$ of Zitácuaro, $S R$ 3707.

11 MEXICO: México: ca. $19 \mathrm{mi}$ SE of Zitácuaro, $S R$ 3716.

22 MEXICO: Sinaloa: Loberas, $R K 10199$.

36 MEXICO: Durango: $10 \mathrm{mi} \mathrm{W}$ of El Salto, $R K 10327$.

MEXICO: Durango: $3 \mathrm{mi} \mathrm{E}$ of Navios, $R K 10364$.

9 MEXICO: Durango: $16 \mathrm{mi} \mathrm{E}$ of Navios, $R K 10344$. Sinaloa: $5 \mathrm{mi} \mathrm{NE}$ of Loberas, $R K 10226$.

9+1B MEXICO: Morelos: $6.5 \mathrm{mi} \mathrm{E}$ of Jiutepec, $S R 3690$.

9 MEXICO: Durango: $16 \mathrm{mi} \mathrm{E}$ of Navios, $R K 10345$.

9 MEXICO: Durango: $18 \mathrm{mi}$ SW jet rtes 30 \& $45, R K$ 10399.

27 MEXICO: Sinaloa: Mazatlán, $R K 10170$.

9 MEXICO: Durango: $5 \mathrm{mi} \mathrm{W}$ of Durango, $R K 10338$.

9 MEXICO: Nuevo León: $21 \mathrm{mi} \mathrm{W}$ of Linares, $R K$ $10470 \mathrm{~A}$.

6 MEXICO: Durango: $2 \mathrm{mi} \mathrm{SW}$ of turnoff to Villa Carranza, $R K 10385$.

6 MEXICO: Nuevo León: ca. $20 \mathrm{mi} \mathrm{S}$ of Monterrey, $R K$ 10449.

8 MEXICO: Coahuila: W of Saltillo, $R K$ 10421. Nuevo León: ca. $20 \mathrm{mi} \mathrm{S}$ of Monterrey, $R K 10431$.

8 MEXICO: Nuevo León: $18 \mathrm{mi} \mathrm{W}$ of Linares, $R K$ 10459.

9 MEXICO: Nuevo León: $18 \mathrm{mi} \mathrm{W}$ of Linares, $R K$ 10458.

9 MEXICO: Durango: $5 \mathrm{mi} \mathrm{W}$ of Durango, $R K 10337$.

18 MEXICO: Nuevo León: $21 \mathrm{mi}$ W of Linares, $R K 10468$.

MEXICO: Durango: $16 \mathrm{mi}$ E of Navios, $R K$ 10346; 2 mi SW of turnoff to Villa Carranza, $R K 10383$.

9 MEXICO: Coahuila: W of Saltillo, $R K 10420$.

6. MEXICO: Durango: $10 \mathrm{mi} \mathrm{W}$ of El Salto, $R K 10325$.

14 MEXICO: Michoacán: ca. $8 \mathrm{mi}$ SE of Zitácuaro, $S R$ 3708.

14 MEXICO: México: ca. $19 \mathrm{mi}$ SE of Zitácuaro, $S R$ 3715.

14 MEXICO: Sinaloa: Loberas, $R K 10204$. 
TABLE 1. Continued

\begin{tabular}{crrr}
\hline \hline Taxon & Gametic chromosome number & Voucher \\
\hline **Gnaphalium stramineum H.B.K. & $14 \quad$ MEXICO: Durango: 5 mi W of Las Adjuntas, RK \\
& & 10303; 11 mi W of Llano Grande, $R K$ 10329.
\end{tabular}

\section{HELIANTHEAE}

Aldama dentata LaLave \& Lex.

Bidens laevis (L.) B.S.P.

Bidens pilosa $\mathrm{L}$.

Bidens pilosa $\mathrm{L}$.

${ }^{+}$Bidens reptans (L.) G. Don var. urbanii (Greenm.)

O. E. Schulz

12

Coreopsis tinctoria Nutt.

Cosmos bipinnatus Cav.

Desmanthodium fruticosum Greenm.

Dracopis amplexicaulis (Vahl.) Cass.

Eclipta alba (L.) Hassk.

Engelmannia pinnatifida Nutt.

Guardiola rosei Robins.

Guardiola thompsonii Van Fassen

Melampodium americanum $\mathbf{L}$.

Melampodium cupulatum A. Gray

Melampodium divaricatum (Rich. in Pers.) DC. Melampodium microcephalum Less.

Melampodium nutans Stuessy

Melampodium pilosum Stuessy

**Melampodium repens Sessé \& Moc.

**Melampodium tepicense Robins.

Melanthera nivea (L.) Small

Milleria quinqueflora $\mathrm{L}$.

**Montanoa pyramidata Sch. Bip.

Parthenice mollis (A. Gray) A. Gray

Parthenium hysterophorus L.

**Perymenium buphthalmoides DC. var. tenellum

(A. Gray) McVaugh

+Perymenium mendezii DC. var. mendezii

Ratibida columnifera (Nutt.) Woot. \& Standl.

Rudbeckia laciniata $\mathrm{L}$.

Sanvitalia ocymoides DC.

Simsia calva (A. Gray \& Engelm.) A. Gray

13

12

18

16

11

9

12
17 MEXICO: Tamaulipas: ca. $5 \mathrm{mi} \mathrm{N}$ of Aldama, $S R$ 3630.

12 MEXICO: Sonora: Imuris, $R K 10141$.

36 MEXICO: Durango: $1 \mathrm{mi}$ E of El Diablo, $R K 10248$.

12 MEXICO: Durango: $1 \mathrm{mi} \mathrm{NE}$ of turnoff to Villa Carranza, $R K$ 10390. Nuevo León: ca. $20 \mathrm{mi} \mathrm{S}$ of Monterrey, $R K 10451$.

MEXICO: Colima: $11.1 \mathrm{mi} \mathrm{S}$ of Colima, SR 3768. 10331 .

MEXICO: Michoacán: $9.7 \mathrm{mi} \mathrm{S}$ of Uruapan, $S R$ 3749.

10 MEXICO: Veracruz: $4 \mathrm{mi} \mathrm{S}$ of Villa Cuauhtemoc, $S R$ $3636 ; 8.5 \mathrm{mi} \mathrm{N}$ of Palma Sola, $S R 3650$.

10 MEXICO: Sonora: $16.3 \mathrm{mi}$ SE of Navajoa, Keil \& Canne 8731.

MEXICO: Sinaloa: $6.9 \mathrm{mi} \mathrm{E}$ of Chilillos, $S G 3052$.

MEXICO: Sinaloa: $5.6 \mathrm{mi}$ E of Chilillos, $S G 3051$.

MEXICO: Colima: $11.1 \mathrm{mi}$ S of Colima, SR 3763.

MEXICO: México: ca. $15 \mathrm{mi} \mathrm{SE}$ of Zitácuaro, $S G$ 3119.

MEXICO: D. F.: La Cima, Anderson 3515 (KSC). ${ }^{\mathrm{f}}$

MEXICO Nayarit: $8.1 \mathrm{mi} \mathrm{E}$ of jct rte $15 \&$ rd to Jalcocotan, $S G 3062$.

MEXICO: Veracruz: $8 \mathrm{mi} \mathrm{S}$ of Cerro Azul, $S R$ 3642; $25 \mathrm{mi} \mathrm{S}$ of Gutiérrez Zamora, SR 3649.

MEXICO: Nayarit: ca. $0.2 \mathrm{mi} \mathrm{N}$ of Tepic, $S G 3058 B$.

MEXICO: México: ca. $5 \mathrm{mi} \mathrm{N}$ of Ixtapan de la Sal, $S R$ 3699.

MEXICO: Sinaloa: $1.3 \mathrm{mi}$ E of Cofradía, $S G 3032$. Sonora: $8.4 \mathrm{mi} \mathrm{S}$ jct rte 15 \& rd to Querobabi, $S G 3007$.

MEXICO: Nuevo León: ca. $20 \mathrm{mi}$ S of Monterrey, $R K$ 10447. Sinaloa: 2 mi E jct rtes $15 \& 40, R K 10163$.

MEXICO: Durango: $2 \mathrm{mi} \mathrm{W}$ of Cruz de Piedra, $R K$ 10376.

MEXICO: Durango: $5 \mathrm{mi} \mathrm{W}$ of Durango, $R K 10333$.

UNITED STATES: Oklahoma: Pittsburg Co.: $2.5 \mathrm{mi}$ $\mathrm{N}$ of Canadian River on rte 69, $R K 10062$.

UNITED STATES: Arizona: Graham Co.: Pinaleño Mts, Wet Canyon, $R K 10085$.

MEXICO: Nuevo León: $26 \mathrm{mi} \mathrm{W}$ of Linares, $R K$ 10473.

MEXICO: Nuevo León: $18 \mathrm{mi} \mathrm{W}$ of Linares. $R K$ 10456. 
TABle 1. Continued

\begin{tabular}{|c|c|c|}
\hline Taxon & \multicolumn{2}{|c|}{ Gametic chromosome number } \\
\hline Simsia eurylepis S. F. Blake & 17 & $\begin{array}{l}\text { MEXICO: Tamaulipas: } 8.1 \mathrm{mi} \mathrm{NE} \text { of Manuel, } S R \\
\text { 3635. }\end{array}$ \\
\hline Simsia foetida (Cav.) S. F. Blake & 17 & MEXICO: Jalisco: $\mathrm{S}$ limits of Atenquique, $S G 3088$. \\
\hline Spilanthes americana (Mut.) Hieron. & 26 & $\begin{array}{l}\text { MEXICO: Veracruz: ca. } 38 \mathrm{mi} \mathrm{S} \text { of Villa } \mathrm{Cu} \\
\quad \text { SR } 3640 .\end{array}$ \\
\hline Tithonia tubaeformis (Jacq.) Cass. & 17 & MEXICO: Puebla: ca. $5 \mathrm{mi} \mathrm{S}$ of Atlixco, $S R 3681$. \\
\hline & 1 & $\begin{array}{l}\text { MEXICO: Sinaloa: } 32 \mathrm{mi} \mathrm{E} \text { of jet rtes } 15 \& 40, R K \\
\text { 10184. Tamaulipas: } 8.1 \mathrm{mi} \mathrm{NE} \text { of Manuel, } S R \\
\text { 3633. }\end{array}$ \\
\hline Trigonospermum melampodioides DC. & 15 & $\begin{array}{l}\text { MEXICO: Michoacán: } 21.9 \mathrm{mi} \text { W of Ciudad Hidalgo, } \\
\text { SR 3731. }\end{array}$ \\
\hline${ }^{+}$Verbesina crocata (Cav.) Less. & 17 & $\begin{array}{l}\text { MEXICO: Puebla: } 10.8 \mathrm{mi} \text { NNW of Tuzantlán, } S R \\
\text { 3685. }\end{array}$ \\
\hline Verbesina encelioides (Cav.) A. & 17 & MEXICO: Sinaloa: Mazatlán, $R K 10169$. \\
\hline Verbesina helianthoides Michx. & 17 & $\begin{array}{l}\text { UNITED STATES: Kentucky: Caldwell Co.: } 5 \mathrm{mi} \mathrm{E} \\
\text { of Princeton, } R K 10022 \text {. }\end{array}$ \\
\hline *Verbesina tequilana Coleman & 17 & $\begin{array}{l}\text { MEXICO: Nayarit: } 8.1 \mathrm{mi} \mathrm{E} \text { of jet rte } 15 \& \mathrm{rd} \text { to } \\
\text { Jalcocotán, } S G 3063 \mathrm{~A} \text {. }\end{array}$ \\
\hline $\begin{array}{l}\text { *Viguiera cordifolia A. Gray var. } \\
\text { Greene }\end{array}$ & 17 & go: $3 \mathrm{mi} \mathrm{E}$ of Navios, $R K 10363$. \\
\hline Viguiera dentata (Cav.) Spreng. & 17 & $\begin{array}{l}\text { MEXICO: Durango: } 18 \mathrm{mi} \mathrm{SW} \text { of jc } \\
\text { 10401. Morelos: } 6.5 \mathrm{mi} \mathrm{E} \text { of } \mathrm{J}\end{array}$ \\
\hline Viguiera ludens (Shinners) M. C. Johnston & 17 & $\begin{array}{l}\text { MEXICO: Coahuila: } 9.4 \mathrm{mi} \mathrm{S} \text { of Rancho Los Charcos, } \\
\quad K 8163 \mathrm{~A} \text {. }\end{array}$ \\
\hline Viguiera stenoloba S. F. Blake & 17 & $\begin{array}{l}\text { MEXICO: Coahuila: } 53 \mathrm{mi} \text { E of turnoff to San Pedro, } \\
R K 10419 \text {. Durango: } 28 \mathrm{mi} \mathrm{SW} \text { jct rtes } 30 \& 45, \\
R K 10395 \text {. Tamaulipas: ca. } 33 \mathrm{mi} \mathrm{S} \text { of San Fer- } \\
\text { nando, } S R \text { 3626. }\end{array}$ \\
\hline dea A. Gray & 18 & $\begin{array}{l}\text { MEXICO: Chihuahua: } 42.9 \mathrm{mi} \text { W of Chihuahua, } \\
K 8299 \mathrm{~A} \text {. }\end{array}$ \\
\hline Zinnia grandiflora Nutt. & 2 & MEXICO: Durango: jct rtes $45 \& 30, R K 10411$. \\
\hline \multicolumn{3}{|l|}{ HELENIEAE } \\
\hline $\begin{array}{l}\text { Bahia absinthifolia Benth. va } \\
\text { A. Gray }\end{array}$ & & $\begin{array}{l}\text { UNITED STATES: Texas: Brewster Co.: } 71.9 \mathrm{mi} \mathrm{S} \\
\text { of Alpine, Stuessy \& Stuessy 2084A. }\end{array}$ \\
\hline nomala Robins. & 1 & $\begin{array}{l}\text { MEXICO: Tamaulipas: } 16.6 \mathrm{mi} \mathrm{SE} \text { of jet rtes } 101 \& \\
180, S R 3628 \text {. }\end{array}$ \\
\hline f. floridana J. R. Johnston & 1 & $\begin{array}{l}\text { UNITED STATES: Texas: Kenedy Co.: } 6.7 \mathrm{mi} \mathrm{N} \text { of } \\
\text { Norias, } S R 3624 \text {. }\end{array}$ \\
\hline a robusta Rose & 1 & MEXICO: Colima: $10.2 \mathrm{mi} \mathrm{S}$ of Colima, $S R 3759$. \\
\hline Florestina liebmanni Sch. Bip. ex Greenm. & 10 & $\begin{array}{l}\text { MEXICO: Veracruz: } 8.5 \mathrm{mi} \mathrm{N} \text { of Palma Sola, } S R \\
\text { 3652. }\end{array}$ \\
\hline Helenium amarum (Raf.) Rock & 1 & $\begin{array}{l}\text { UNITED STATES: Kentucky: Marshall Co.: } 2 \mathrm{mi} \mathrm{E} \\
\text { of jet rtes } 299 \& 80, R K 10024 \text {. }\end{array}$ \\
\hline *Helenium apterum (Blake) Bierner & 17 & $\begin{array}{l}\text { MEXICO: Durango: } 1 \mathrm{mi} \mathrm{W} \text { of Las Adjuntas, } R K \\
\text { 10306. }\end{array}$ \\
\hline egans DC. var. amphibolum (A. C & & MEXICO: Nuevo León: ca. $20 \mathrm{mi} \mathrm{S}$ of Monterrey, $R K$ \\
\hline Hele & 1 & $\begin{array}{l}\text { 10445. } \\
\text { MEXICO: Sonora: ca. } 9 \mathrm{~km} \mathrm{NW} \text { of Vicam, } R K 10147 \text {. }\end{array}$ \\
\hline Helenium quadridentatum Labill. & 1 & $\begin{array}{l}\text { MEXICO: Michoacán: } 13 \mathrm{mi} \text { W of jet rtes } 37 \& 120 \text {, } \\
\text { SR } 3740 \text {. }\end{array}$ \\
\hline $\begin{array}{l}\text { Hymenopappus scabiosaeus L'Her. var. corymbosu } \\
\text { (T. \& G.) B. L. Turner }\end{array}$ & 1 & $\begin{array}{l}\text { UNITED STATES: Texas: Sutton Co.: ca. } 3 \mathrm{mi} \text { E of } \\
\text { Sonora, } R K 10070 \text {. }\end{array}$ \\
\hline Hymenoxys odorata DC. & 1 & $\begin{array}{l}\text { UNITED STATES: Texas: Sutton Co.: ca. } 3 \mathrm{mi} \text { E of } \\
\text { Sonora, } R K 10073 \text {. }\end{array}$ \\
\hline $\begin{array}{l}\text { Palafoxia rosea (Bush.) Cory var. robusta } \\
\text { (Rydb.) Cory }\end{array}$ & 11 & MEXICO: Veracruz: $7.3 \mathrm{mi} \mathrm{N}$ of Palma Sola, $S R 3653$. \\
\hline $\begin{array}{l}\text { Perityle crassifolia Brandg. var. robusta (Rydb.) } \\
\text { Everly }\end{array}$ & 1 & $\begin{array}{l}\text { MEXICO: Baja California Sur: } 4.8 \mathrm{mi} \mathrm{NE} \text { of La Paz, } \\
\text { SG } 3045 \text {. }\end{array}$ \\
\hline Perityle microglossa Benth. var. microglossa & 34 & $\begin{array}{l}\text { MEXICO: Sonora: ca. } 10 \mathrm{~km} \mathrm{~N} \text { of Cd Obregon, } R K \\
\text { 10150. Sinaloa: Mazatlán, RK } 10156 .\end{array}$ \\
\hline $\begin{array}{l}\text { Perityle tu } \\
{ }^{+} \text {Pseudoclap }\end{array}$ & $\begin{array}{l}17 \\
19\end{array}$ & $\begin{array}{l}\text { MEXICO: Durango: } 1 \mathrm{mi} \mathrm{E} \text { of El Diablo, } R K 10247 . \\
\text { UNITED STATES: New Mexico: Sandoval Co.: ca. } 3 \\
\text { mi N of San Ysidro } K 10879\end{array}$ \\
\hline
\end{tabular}


TABle 1. Continued

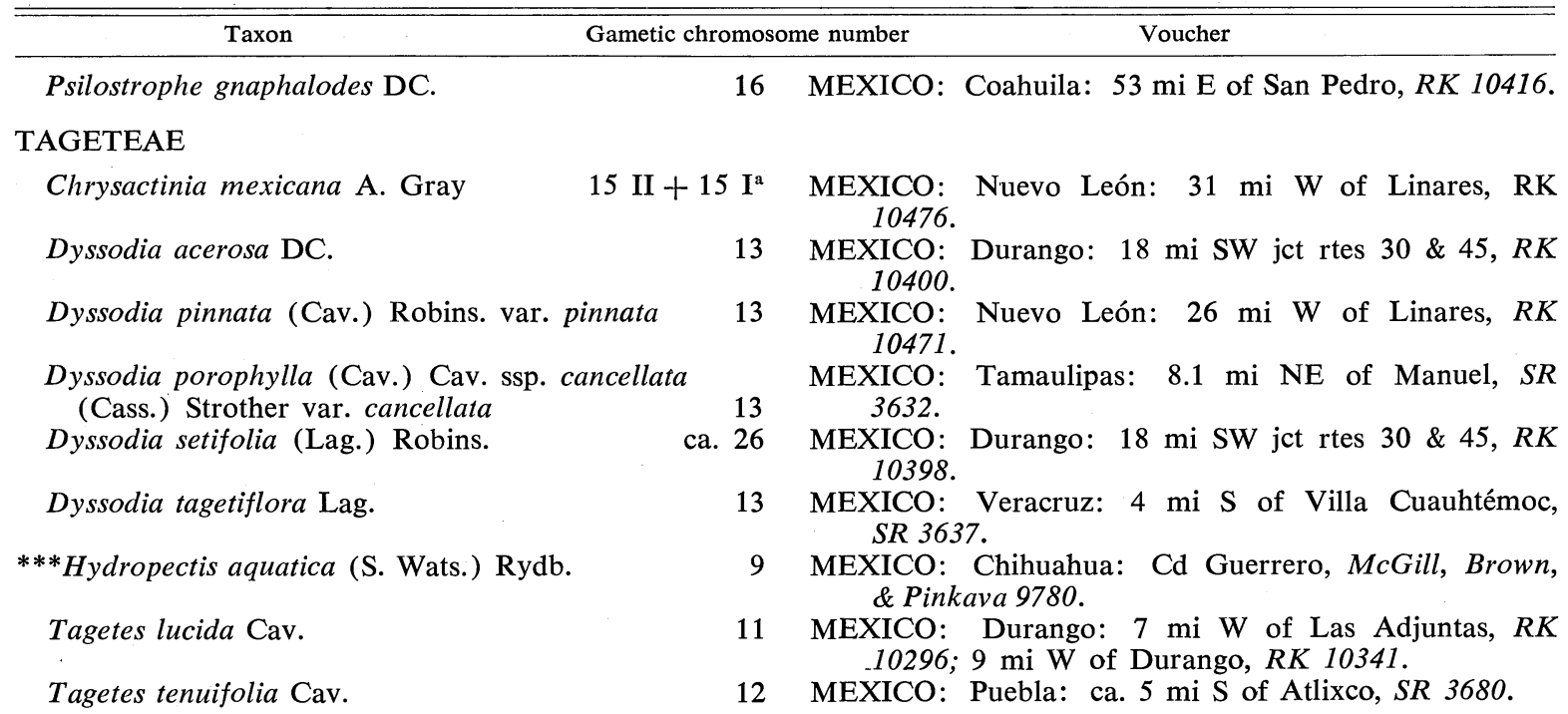

\section{ANTHEMIDEAE}

Achillea lanulosa Nutt.

Anthemis arvensis L.

$36 \mathrm{I}^{\mathrm{a}}$ MEXICO: Durango: $10 \mathrm{mi}$ W of E1 Salto, $R K 10324$.

9 UNITED STATES: Missouri: Mississippi Co.: $1.5 \mathrm{mi}$ W of Mississippi River on rte 80, RK 10029.

\section{SENECIONEAE}

Odontotrichum sinuatum (Cerv.) Rydb.

**Senecio arizonicus Greene

Senecio douglasii DC. var. longilobus (Benth.) L. Benson

Senecio fendleri A. Gray

${ }^{* *}$ Senecio flaccidus Less.

Senecio glabellus Poir.

Senecio imparipinnatus Klatt

Senecio multilobatus T. \& G. ex A. Gray

Senecio runcinatus Less.

Senecio cf. toluccanus DC.

Senecio tridenticulatus Rydb.

**Senecio wootonii Greene

MEXICO: Durango: $11 \mathrm{mi} \mathrm{W}$ of Llano Grande, $R K$ 10328.

MEXICO: Durango: $6 \mathrm{mi} \mathrm{E}$ of La Ciudad, $R K 10289$. UNITED STATES: Arizona: Coconino Co.: Oak Creek Canyon, $K 10795$.

UNITED STATES: Arizona: Coconino Co.: $13 \mathrm{mi} \mathrm{S}$ of The Gap, $K$ 10803. New Mexico: Sandoval Co.: Albuquerque, $K 10724$.

UNITED STATES: Colòrado: Fremont Co.: Royal Gorge, $K 10702$.

MEXICO: Durango: $7 \mathrm{mi} \mathrm{W}$ of Las Adjuntas, $R K$ 10299.

UNITED STATES: Missouri: Mississippi Co.: $1.5 \mathrm{mi}$ W of Mississippi River on rte 80, RK 10027.

UNITED STATES: Texas: Jefferson Co.: Port Arthur, Somers \& Keil 10627.

UNITED STATES: Arizona: Coconino Co.: Oak Creek Canyon, $K$ 10798, $10 \mathrm{mi} \mathrm{E}$ of Jacob Lake, $K$ 10814. Utah: Grand Co.: Arches Natl Park, near Devil's Garden, $K 10851$.

UNITED STATES: Arizona: Graham Co.: Pinaleño Mts., $K$ 10790; Coconino Co.: $10 \mathrm{mi} \mathrm{S}$ of Mormon Lake, $K 10800$.

MEXICO: Veracruz: $0.8 \mathrm{mi} \mathrm{NW}$ of La Joya, SR 3674. MEXICO: Durango: $10 \mathrm{mi} \mathrm{SW}$ of La Ciudad, $R K$ 10273; $5 \mathrm{mi} \mathrm{W}$ of Las Adjuntas, $R K 10304$.

UNITED STATES: Colorado: Fremont Co.: Royal Gorge, $K$ 10703. Kit Carson Co.: Near Seibert, K 10697.

UNITED STATES: Arizona: Cochise Co.: Chiricahua Mts, Rustler's Park, K 10760; Graham Co.: Pinaleño Mts, Heliograph Peak, K 10791.

\footnotetext{
${ }^{\mathrm{a}}$ Sporophytic chromosome number; ${ }^{\mathrm{b}} \mathrm{RK}=$ Roberts \& Keil; ${ }^{\mathrm{c}} \mathrm{SR}=$ Stuessy \& Roberts; ${ }^{\mathrm{a}} \mathrm{K}=$ Keil; ${ }^{\mathrm{e}} \mathrm{SG}=$ Stuessy \& Gardner; ${ }^{f}$ counted by L. C. Anderson $(2 n=54)$.

* First report for variety; ** species; *** genus.

+ New reported number for taxon.
} 
as $n=18$ (Turner, Ellison, and King, 1961; Turner, Powell, and King, 1962). Our new count of $n=17$ indicates possible aneuploidy in the species, and suggests continued study of other populations.

Viguiera cordifolia has been reported previously as $n=17$ (Heiser and Smith, 1955) from the typical variety; our count of the same number is from var. latisquama as recognized by Blake (1913). Viguiera ludens (= Helianthus ludens Shinners), $n=17$, counted previously by Powell and Sikes (1970) and Yates (1971), has until now been collected only from the type vicinity in Culberson County, Texas. Our voucher was obtained ca. $500 \mathrm{~km}$ away in southwestern Coahuila. At the type locality, $V$. ludens grows as a weed in a cotton field. Our report supports the suggestion of Heiser (1969) that $V$. ludens probably is a Mexican species only recently introduced into Texas.

Helenieae-Helenium apterum $(n=17$, first reported here) and $H$. scorzoneraefolium (DC.) A. Gray $(n=17$; Bierner, 1972) are the only two species of sect. Hecubaea, regarded by Bierner (1972) as the most primitive section in the genus. Although three of the five sections in Helenium have aneuploid series, sect. Hecubaea is chromosomally uniform.

Our count of $n=12$ for Hymenoxys odorata is of interest because of the recent reports of descending aneuploidy $(n=15,14,12,11)$ in the species (Sanderson and Strother, 1973). The previous counts of $n=12$ have come from populations near Del Rio in Val Verde County, Texas. Our count from adjacent Sutton County extends the known range of the cytotype in this general region.

Pseudoclappia arenaria is known from previous counts as $n=18 \pm 1$ (Powell and Turner, 1963) and $n=18$ or 19 (Reveal and Spellenberg, 1976). Our count of $n=19$ establishes this as a firm level for the genus.

Tageteae-Rydberg (1915-16) divided the Tageteae into two subtribes, the Pectidinae and the Tagetinae. The Pectidinae comprised Pectis and Hydropectis; the remaining genera of the tribe were placed into the Tagetinae. Our count of $n=9$ for Hydropectis aquatica is the first report for the genus, and also is the first report of $n=9$ for any member of the Tageteae. This chromosome number is closer to the base numbers of Dyssodia $(x=7,8,13)$, Urbinella $(x=$ $8)$, and Nicolletia $(x=10)$ of the Tagetinae than to that of Pectis $(x=12)$. On the basis of morphological and physiological evidence both the senior author and Dr. J. L. Strother (personal communication) have concluded that Hydropectis would be better placed in the Tagetinae than in the Pectidinae.
Senecioneae-The monotypic genus, Pippenalia $\mathrm{McVaugh}$, was established recently (McVaugh, 1972) to include Odontotrichum delphinifolium Rydb. This species was treated by Pippen (1968) in his revision of Odontotrichum as an excluded taxon of doubtful affinities but which (p. 435) ". . . should probably be referred to the Heleneae [sic]." McVaugh (1972) continued this discussion (p. 470) with the statement that: "The correct taxonomic position of the genus is still open to question. In habit the plants are very like those of what Pippen has called the 'cacalioid' members of the Senecioneae; the styles are at least not unlike the Senecioid type; the plump naked achenes suggest those of some members of the Heliantheae, but are unusual if not unique among the North American Senecioneae." Robinson and Brettell (1973) placed Pippenalia in the Senecioneae near Psacalium Cass. and Psacaliopsis $\mathbf{H}$. Robins. \& Brettell, but Nordenstam (in press) regards the genus as closest to Senecio of the same tribe. Chromosomally, species of Odontotrichum and Psacalium are known as $n=30$, whereas Senecio has many reported numbers of which $n$ $=20,30$, and 40 are common. Our first report for Pippenalia is $2 n=$ ca. 60 , with a complex meiotic configuration of over 20 bivalents, many univalents, and possibly a few multivalents. Pollen stainability in our voucher is $75 \%$ as shown by a sample of 300 grains in acetocarmine. The meiotic irregularities and reduced pollen stainability suggest a possible hybrid origin of Pippenalia. This is an interesting possibility because, being monotypic, Pippenalia could have originated from an intergeneric cross. More populations of this species obviously need to be found and examined cytologically.

\section{LITERATURE CITED}

BIERNER, M. W. 1972. Taxonomy of Helenium sect. Tetrodus and a conspectus of North American Helenium (Compositae). Brittonia 24: 331-355.

Blake, S. F. 1913. A revision of the genus Viguiera. Contrib. Gray Herb. Harv. Univ. 54: 1-205.

Bolick, M. R., aND J. J. Skvarla. 1976. A reappraisal of the pollen ultrastructure of Parthenice mollis Gray (Compositae). Taxon 25: 261-264.

Cave, M. (ed.). 1958-65. Index to plant chromosome numbers, 1956-64 + Suppl. Univ. North Carolina Press, Chapel Hill.

Darlington, C. D., and A. P. Wylie. 1955. Chromosome atlas of flowering plants. ed. 2. George Allen and Unwin, Ltd., London.

DeJong, D. C. D., AND E. K. Longpre. 1963. Chromosome studies in Mexican Compositae. Rhodora 65: $225-240$.

Fedorov, A. A. (ed.). 1969. Khromosomnye Chisla Tsvetkovykh Rasteny (Chromosome numbers of flowering plants). Acad. Sci. U.S.S.R., Leningrad.

Heiser, C. B., JR. 1969. The North American sunflowers (Helianthus). Mem. Torr. Bot. Club 22 (3): 1-218. 
AND D. M. SMITH. 1955. New chromosome numbers in Helianthus and related genera (Compositae). Proc. Indiana Acad. Sci. 64: 250-253.

HofFMANN, O. 1890-94. Compositae, p. 87-391. In A. Engler \& K. Prantl, Die natürlichen Pflanzenfamilien, vol. 4(5). Leipzig.

Keil, D. J., AND D. J. Pinkava. 1976. Chromosome counts and taxonomic notes for Compositae from the United States and Mexico. Amer. J. Bot. 63: 1393-1403.

—, AND T. F. Stuessy. 1975. Chromosome counts of Compositae from the United States, Mexico, and Guatemala. Rhodora 77: 171-195.

KING, R. M., AND H. Robinson. 1970. Studies in the Eupatorieae (Compositae). XIX. New combinations in Ageratina. Phytologia 19: 208-229.

McVaugh, R. 1972. Compositarum Mexicanarum pugillus. Contrib. Univ. Mich. Herb. 9: 359-484.

Moore, R. J. (ed.). 1970-74. Index to plant chromosome numbers for 1968-72. Regnum Veg. 68:1115; 77: 1-112; 84: 1-134; 90: 1-539; 91: 1-108.

NordenstaM, B. In press. Systematics of Senecioneae s. lat. In V. H. Heywood, J. B. Harborne, and B. L. Turner (eds.), The biology and chemistry of the Compositae. Academic Press, N.Y.

ORNDUFF, R. (ed.). 1967-69. Index to plant chromosome numbers for 1965-67. Regnum Veg. 50: 1-128; 55: 1-126; 59: 1-129.

Payne, W. W., P. H. Raven, and D. W. Kyhos. 1964. Chromosome numbers in Compositae. IV. Ambrosieae. Amer. J. Bot. 51: 419-424.

Pippen, R. W. 1968. Mexican "Cacalioid" genera allied to Senecio (Compositae). Contrib. U.S. Nat. Herb. 34: 365-447.

Powell, A. M., AND S. Sikes. 1970. Chromosome numbers of some Chihuahuan desert Compositae. Southwest. Nat. 15: 175-186.

-, AND B. L. Turner. 1963. Chromosome numbers in the Compositae. VII. Additional species from the southwestern United States and Mexico. Madroño 17: 128-140.

Reveal, J. L., AND R. Spellenberg. 1976. Miscellaneous chromosome counts of western American plants. Rhodora 78: 37-52.

Robinson, B. L. 1926. The woody species of $E u$ patorium and Ophryosporus occurring in Mexico. Contrib. U.S. Nat. Herb. 23: 1432-1470.

Robinson, H., AND R. D. Brettell. 1973. Studies in the Senecioneae (Asteraceae). V. The genera Psacaliopsis, Barkleyanthus, Telanthophora and Roldana. Phytologia 27: 401-439.

Rydberg, P. A. 1915-16. Tribe 11. Tageteae. North Amer. Fl. 34: 147-216.

SANDERson, S. C., AND J. L. Strother. 1973. The origin of aneuploidy in Hymenoxys odorata. Nature-New Biol. 242: 220-221.

SAUCK, J. R. 1975. Distribution, chromosomes, and taxonomy of Parthenice mollis (Compositae). Madroño 23: 227-234.

SHINNERS, L. H. 1949. Revision of the genus Egletes Cassini north of South America. Lloydia 12: 239247.

Solbrig, O. T. 1960a. Cytotaxonomic and evolutionary studies in the North American species of Gutierrezia (Compositae). Contrib. Gray Herb. 188: 3-63.

1960b. The status of the genera Amphipappus, Amphiachyris, Greenella, Gutierrezia, Gymnosperma and Xanthocephalum (Compositae). Rhodora 62: 43-54.

1961. Synopsis of the genus Xanthocephalum (Compositae). Rhodora 63: 151-164.

, D. W. Kyhos, M. Powell, and P. H. Raven. 1972. Chromosome numbers in Compositae VIII: Heliantheae. Amer. J. Bot. 59: 869-878.

Strother, J. L. 1976. Chromosome studies in Compositae. Amer. J. Bot. 63: 247-250.

Stuessy, T. F. 1970. Chromosome studies in Melampodium (Compositae, Heliantheae). Madroño 20: 365-372.

- 1971. Chromosome numbers and phylogeny in Melampodium (Compositae). Amer. J. Bot. 58: 732-736.

. 1972. Revision of the genus Melampodium (Compositae: Heliantheae). Rhodora 74: 1-70, 161-219.

- 1973. A systematic review of the subtribe Melampodiinae (Compositae, Heliantheae). Contrib. Gray Herb. 203: 65-80.

Turner, B. L., J. H. Beaman, and H. F. L. Rock. 1961. Chromosome numbers in the Compositae. V. Mexican and Guatemalan species. Rhodora 63: 121-129.

, W. L. Ellison, AND R. M. KING. 1961. Chromosome numbers in the Compositae. IV. North American species, with phyletic interpretations. Amer. J. Bot. 48: 216-223. , AND D. FLYR. 1966. Chromosome numbers in the Compositae. X. North American species. Amer. J. Bot. 53: 24-33.

, M. Powell, AND R. M. KING. 1962. Chromosome numbers in the Compositae. VI. Additional Mexican and Guatemalan species. Rhodora 64: 251-271.

UrbatsCH, L. E. 1975. First chromosome number reports for some Compositae. Southwest. Nat. 20: 283-285.

YATES, W. F., JR. 1971. In IOPB Chromosome reports XXXII. Taxon 20: 356. 